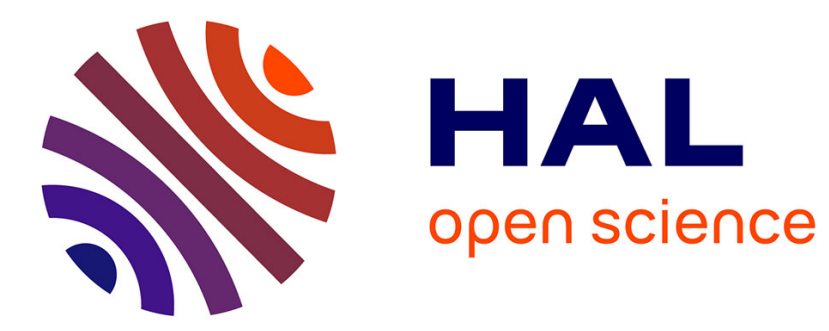

\title{
Quadrotor stabilization under time and space constraints using implicit PID controller
}

\author{
Siyuan Wang, Andrey Polyakov, Gang Zheng
}

\section{To cite this version:}

Siyuan Wang, Andrey Polyakov, Gang Zheng. Quadrotor stabilization under time and space constraints using implicit PID controller. Journal of The Franklin Institute, Elsevier, 2022. hal-03582071

\section{HAL Id: hal-03582071 \\ https://hal.inria.fr/hal-03582071}

Submitted on 21 Feb 2022

HAL is a multi-disciplinary open access archive for the deposit and dissemination of scientific research documents, whether they are published or not. The documents may come from teaching and research institutions in France or abroad, or from public or private research centers.
L'archive ouverte pluridisciplinaire HAL, est destinée au dépôt et à la diffusion de documents scientifiques de niveau recherche, publiés ou non, émanant des établissements d'enseignement et de recherche français ou étrangers, des laboratoires publics ou privés. 


\title{
Quadrotor stabilization under time and space constraints using implicit PID controller
}

\author{
Siyuan Wang ${ }^{1}$, Andrey Polyakov ${ }^{2,3}$, and Gang Zheng ${ }^{2,4}$ \\ ${ }^{1}$ INSA Centre Val de Loire, 18022 Bourges, France \\ ${ }^{2}$ Inria - Lille Nord Europe, 59650 Lille, France \\ ${ }^{3}$ ITMO University, Saint-Petersburg Russia \\ ${ }^{4}$ School of Mathematics and Big Data, Foshan University, Foshan, China
}

\begin{abstract}
In this paper, the quadrotor stabilization under time and state constraints is studied. The objective is to design a nonlinear controller under time and state constraint for quadrotor. The nonlinear quadrotor model is built by the Euler-Lagrange approach while ignoring the Coriolis terms, hub moment and force. Based on quadrotor's dynamic model, a nonlinear feedback controller is designed for the quadrotor stabilization under time and state constraints. This feedback is an implicit PID controller where the feedback gains are obtained from LMIs (Linear matrix inequalities). LMI system characterizing the system stability and convergence properties is built based on convex embedding approach and implicit Lyapunov function method. To demonstrate the application prospects of implicit PID controller, robustness analysis is provided to show the property of implicit PID controller under external disturbance. The key novelty of this paper is that the implicit PID controller is proven feasible for applying to the quadrotor under time and state constraints, which is also the main outcome. Keywords - Nonlinear, robust, quadrotor, constraint
\end{abstract}

\section{Introduction}

In the past several decades, researchers have shown a considerable interest in the quadrotor control. As a vertical take-off and landing unmanned aerial vehicle, quadrotor shows a wide range of applications, such as rescue, delivery, mapping, surveillance, etc. Quadrotor is not only an efficient and flexible aerial vehicle, but also a typical nonlinear and coupled under-actuated system, which motives the researcher to develop more advanced automatic control algorithms.

So far, many researchers have investigated both linear and nonlinear algorithms to control quadrotor. Linear controllers such as proportional-integral-derivative (PID), linear quadratic regulator (LQR) design, and $H_{\infty}$ have been widely applied. In 1, the PID controller is applied to stabilize the attitude of quadrotor. In 2, 3], both attitude and position were stabilized by the PID controller. To optimize the performance of linear controller, LQR 4, 5 has been applied to minimize the cost function of error and inputs. $H_{\infty}$ as a robust controller was also investigated in 6], [7. Tuning the parameter of linear controller is another issue to be solved after the controller design. Linear Matrix Inequalities(LMIs), as an advanced approach, is easy to be implemented, which now can be easily solved by MATLAB and many other softwares. Although some linear control methods aforementioned were widely applied, a potential drawback is that they can only provide limit performance for systems with high nonlinearity under large operation space.

In recent years, nonlinear controllers for quadrotor have received much attention, since they could improve the quadrotor performance such as faster movement, higher precision and more robustness. In the literature, different types of nonlinear controllers have been applied on quadrotor such as feedback linearization 6, 8, 9, MPC-based (model predictive control) techniques 10, 11, 12, backstepping approach [13, 14, [15], sliding mode methodology [16, 17], 18]. Generally, feedback linearization allows to apply linear methodology to nonlinear system, however it requires more exact model to avoid the loss of precision due to the linearization process. Backstepping controller is well-known for underactuated system control. Although it could provide a fast convergence and guarantees globally boundedness of tracking error, it is still limited by the explosion of terms. MPC is another nonlinear technique to estimate the future system state while minimizing the error by solving optimal control problems. On-line optimization is the key limit of MPC, which requires relatively high computation power and makes it not appropriate for quadrotor. Sliding mode control has been known as one of the important tools for robust control, since it is able to compensate the non-vanishing perturbation 
by discontinuous controller. However, this discontinuous controller leads to the chattering problem [19. Besides, the gain selection of the nonlinear control methods is another problem to be solved. In [20], the controller design is based on the explicit Lyapunov functions, which makes the gain selection to be a complex nonlinear problem. On the other side, the Implicit Lyapunov function is applied for controller design in 21, and one of the most attractive features of this approach is its simple gain selection procedure, which in fact is equivalent to solve an LMI problem. Therefore we propose in this paper to use this implicit approach to design controller for quadrotor.

Another important issue is related to practical constraints of quadrotor. In practice, many environment restrictions, hardware constraints and different objectives impose severe requirements for quadrotor's movement, e.g. state constraints, input constraints, time constraints, computation constraints, and so on. Due to the physical constraints of actuator, some researchers studied the motion control under input saturation [22, 23. Some others studied the state constraints for practical reason [24], 25. For example, the attitude constraints are important for the application of visual servoing, where the orientation of quadrotor is limited in a small range to keep the target in the camera's field of view. Besides every quadrotor has a limit payload capacity which means there is a trade-off between performance and the flying time. This restricts quadrotor's working region and time, and its computation capacity. Therefore a simpler controller that requires less computation power and reacts faster than a complex one is preferred. Degradation of control precision could happen if the limits of computational power is exceeded. In [26], the authors showed that a quadrotor with backstepping controller consumes 5\% more energy than linear PID controller.

The problem of quadrotor control under constrains is not an easy problem 24]. In the literature, most of the research use PID with saturation, sliding mode, LQR, etc to solve the time, attitude or input constraints of quadrotor. For example in [27], the author studied the problem of quadrotor control under input and state constraints, while the problem of time constraint was not involved. Besides, the inner-outer loop control structure with saturation function, proposed in that paper, may limit its performance. In [28], the path planning of quadrotor under attitude constraints has been investigated, however it did not provide the proof of stability or robustness analysis, and no time constraint was considered. However, th restriction of transient time (i.e., time constraint) could be important in many situations, for example, in the case of trajectory tracking [29, or the formation control of quadrotor [30] and collision avoidance. From the theoretical point of view, the simplest way to fulfill this time constraint is by finite-time stabilization [31, 32. Since the linear algorithms for example LMI-based scheme can be an option as well to be constructed for satisfying state constraints, and the gain design of using Implicit Lyapunov function can be reduced to an LMI as well, therefore, the nonlinear implicit finite-time controller might be a good candidate to achieve the time and state constraints stabilization [33, 34.

Motivated by this thought, this paper proposes an implicit PID controller for quadrotor under time and state constraint. Compared to classical PID-based nonlinear controllers, such as 35], which normally do not consider time and state constraints and assume the precise knowledge of system's model, the proposed controller deal with the robust finite-time quadrotor control problem under time and state constraint. Consequently, those so-called PID-based nonlinear controller cannot be applied to treat the investigated problem of this paper. This paper extends the mentioned control design methodology to nonlinear quadrotor model using convex embedding approach. The preliminary version of this paper has been presented at Europe Control Conference 2019 [36]. The key differences are

- The implicit PD algorithm is extended to implicit PID quadrotor control allowing rejecting constant perturbations of unknown magnitude.

- Robustness analysis is added.

- More simulation examples and comparisons are provided.

- proofs of all claims are given.

The paper is organized as follows: Section 2 presents the notations and definitions used in this paper. The problem statement is introduced in Section 3. In Section 4, the original quadrotor model was simplified and reorganized which makes it possible to use convex embedding method. Section 5 gives the details of designing a nonlinear implicit PID controller which fulfills the time and state constraints. Section 6 provides the robustness analysis for implicit PID controller of quadrotor. Section 7 and 8 present the numerical results and conclusion respectively.

\section{Preliminaries}

\subsection{Notations}

The following notations will be used in this paper. 
- $\mathbb{R}$ : the set of real numbers, and $\mathbb{R}_{+}=\{x \in \mathbb{R}: x>0\}$.

- $\|\cdot\|$ : the Euclidian norm in $\mathbb{R}^{n} ;\|x\|_{p}:=x^{\top} P x$.

- $\operatorname{diag}\left\{\lambda_{i}\right\}_{i=1}^{n}$ : the diagonal matrix with elements $\lambda_{i}$

- $P \leq 0(\geq 0,<0,>0)$ for symmetric $P \in \mathbb{R}^{n \times n}$ represent $P$ is semi-negative(positive) definite and negative (positive) definite.

- $\lambda_{\min }(P)$ : minimal eigenvalue of $\mathrm{P} ; \lambda_{\max }(P)$ : maximal eigenvalue of $P$.

- For $P \geq 0$ the square root of $P$ is defined by a matrix $P^{\frac{1}{2}}=Q$ such that $Q^{2}=P$.

- A continuous function $\sigma: \mathbb{R}_{+} \rightarrow \mathbb{R}_{+}$belongs to the class $\mathcal{K}$ if it is monotone increasing and $\sigma(h) \rightarrow 0^{+}$as $h \rightarrow 0^{+}$.

\subsection{Definitions}

Consider the following general form of system

$$
\dot{x}=f(t, x), \quad x(0)=x_{0}
$$

where $f: \mathbb{R}_{+} \times \mathbb{R}^{n}$ is the nonlinear vector field and $x \in \mathbb{R}^{n}$ is the state of system.

Definition 1 ([33], [37], [38]) The origin of system is said to be globally finite-time stable if:

1. Lyapunov stability: $\exists \delta \in \mathcal{K}$ such that $\left.\| x\left(t, x_{0}\right)\right) \| \leq \delta\left(\left\|x_{0}\right\|\right)$ for all $x_{0} \in \mathbb{R}^{n}, t \in \mathbb{R}_{+}$.

2. Finite-time attractivity: there exists a locally bounded function $\mathfrak{T}: \mathbb{R}^{n} \backslash\{0\} \rightarrow \mathbb{R}_{+}$, such that for all $x_{0} \in \mathbb{R}^{n} \backslash\{0\}$, any solution $x\left(t, x_{0}\right)$ of system is defined at least on $\left[0, \mathfrak{T}\left(x_{0}\right)\right)$ and $\lim _{t \rightarrow \mathfrak{T}\left(x_{0}\right)} x\left(t, x_{0}\right)=0$.

The function $\mathfrak{T}$ is called the settling time function of system.

Theorem 1 [33] If there exists a continuous function

$$
\begin{array}{r}
Q: \mathbb{R}_{+} \times \mathbb{R}^{n} \rightarrow \mathbb{R} \\
(V, x) \rightarrow Q(V, x)
\end{array}
$$

satisfying the following conditions

C1) $Q$ is continuous and differentiable outside the origin;

C2) $\forall x \in \mathbb{R}^{n} \backslash\{0\}, \exists V \in \mathbb{R}_{+}$such that

$$
Q(V, x)=0
$$

C3) let $\Omega=\left\{(V, x) \in \mathbb{R}_{+} \times \mathbb{R}^{n}: Q(V, x)=0\right\}$ and

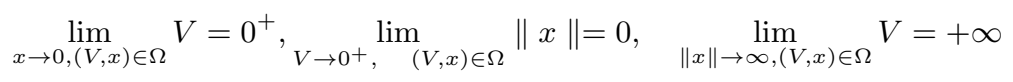

C4) $\frac{\partial Q(V, x)}{\partial V}<0$ for all $V \in \mathbb{R}_{+}$and $x \in \mathbb{R}^{n} \backslash\{0\}$;

C5) There exist $c>0$ and $0<\mu \leq 1$ such that

$$
\sup _{t \in \mathbb{R}_{+}} \frac{\partial Q(V, x)}{\partial x} f(t, x) \leq c V^{1-\mu} \frac{\partial Q(V, x)}{\partial V}
$$

for all $(V, x) \in \Omega$

then the origin of the system is globally uniformly finite-time stable and $\mathfrak{T}\left(x_{0}\right) \leq \frac{V_{0}^{\mu}}{c \mu}$, where $Q(V, x)=$ 0 .

\section{Problem statement}

In the literature, the dynamic model of quadrotor has been well established [39], [40]. In this paper, the quadrotor model ignores the hub moment and force which are relative smaller comparing with actuator's forces and moments [41].

Two different coordinate systems inertial and body frames, denoted by $i$ and $b$ respectively are used for the quadrotor modeling( Fig 1). The position in inertial frame of quadrotor are given by $(x, y, z)$ and quadrotor attitude is defined by Euler angle pitch-roll-yaw $(\theta, \phi, \psi)$.

The total thrust $u_{1}$ defined in body frame of quadrotor is the sum of four thrusts $f_{1}, f_{2}, f_{3}, f_{4}$ produced by four propellers, $u_{1}=f_{1}+f_{2}+f_{3}+f_{4}$. The thrust of each propeller is denoted as 


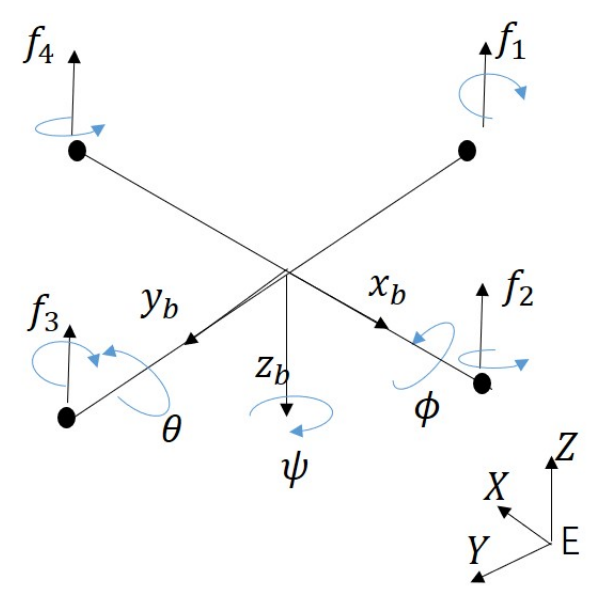

Figure 1: Quadrotor Coordinate System

$f_{i}=k \omega_{i}^{2}$, with thrust coefficient $k$ and rotation speed of propeller $\omega_{i}$. The thrust force, described in inertial frame, can be obtained by $R(\phi, \theta, \psi) \cdot \boldsymbol{F}$ with $\boldsymbol{F}=\left[0,0, u_{1}\right]^{T}$ and

$$
R(\phi, \theta, \psi)=\left[\begin{array}{ccc}
C_{\theta} C_{\psi} & -S_{\phi} S_{\theta} C_{\psi}+C_{\phi} S_{\psi} & -C_{\phi} S_{\theta} C_{\psi}-S_{\phi} S_{\psi} \\
-C_{\theta} S_{\psi} & S_{\phi} S_{\theta} S_{\psi}+C_{\phi} C_{\psi} & C_{\phi} S_{\theta} S_{\psi}-S_{\phi} C_{\psi} \\
S_{\theta} & S_{\phi} C_{\theta} & C_{\phi} C_{\theta}
\end{array}\right]
$$

with notations $C_{\theta}=\cos \theta$ and $S_{\theta}=\sin \theta$, etc.

$\boldsymbol{q}=(x, y, z, \phi, \theta, \psi)^{T}$ The Euler-Lagrange equation with external generalized forces is

$$
\frac{d}{d t}\left(\frac{\partial L}{\partial \dot{\boldsymbol{q}}}\right)-\frac{\partial L}{\partial \boldsymbol{q}}=\left[\begin{array}{c}
R(\phi, \theta, \psi) \boldsymbol{F} \\
\boldsymbol{\tau}
\end{array}\right]
$$

where $\boldsymbol{\tau}$ is the moments of roll,pitch and yaw. Denote $\xi=(x, y, z)^{T}$ and $\eta=(\phi, \theta, \psi)^{T}$. The Lagrangian is defined as

$$
L(\boldsymbol{q}, \dot{\boldsymbol{q}})=T_{\text {trans }}+T_{\text {rot }}-P
$$

where $T_{\text {trans }}=\frac{1}{2} m \dot{\boldsymbol{\xi}}^{T} \dot{\boldsymbol{\xi}}$ is quadrotor's translational kinetic energy, and $T_{\text {rot }}=\frac{1}{2} \boldsymbol{\omega}^{T} I \boldsymbol{\omega}$ is quadrotor's rotational kinetic energy where $I$ is the inertia matrix and $\boldsymbol{\omega}=(p, q, r)$ is the body axis rotational velocities. $P=m g z$ represents potential energy of quadrotor, where $m$ is the quadrotor mass, and $g$ is the gravity acceleration.

$\boldsymbol{\omega}$ and $\dot{\eta}$ have the following relation

$$
\omega=\left[\begin{array}{ccc}
1 & 0 & -\sin \theta \\
0 & \cos \phi & \sin \phi \cos \theta \\
0 & -\sin \phi & \cos \phi \cos \theta
\end{array}\right] \dot{\eta}={ }^{B} W_{l} \dot{\eta}
$$

Remark that when the $\phi, \theta$ angle is smaller, the matrix ${ }^{B} W_{l}$ is approximated to 1 , then there will be $\boldsymbol{\omega} \approx \dot{\boldsymbol{\eta}}$. In most of the research article, the author uses this approximation to simplify the dynamic model 42 .

Define

where

$$
\boldsymbol{J}(\phi, \theta)={ }^{B} W_{l}^{T} I^{B} W_{l}
$$

$$
I=\left[\begin{array}{ccc}
I_{x x} & 0 & 0 \\
0 & I_{y y} & 0 \\
0 & 0 & I_{z z}
\end{array}\right]
$$

Therefore

$$
T_{\text {rot }}=\frac{1}{2} \dot{\eta}^{T} \boldsymbol{J} \dot{\eta}
$$

Finally the Lagrangian is

$$
L(\boldsymbol{q}, \dot{\boldsymbol{q}})=\frac{1}{2} m \dot{\boldsymbol{\xi}}^{T} \dot{\boldsymbol{\xi}}+\frac{1}{2} \dot{\eta}^{T} \boldsymbol{J} \dot{\eta}-m g z
$$

Since the Lagrangian term $L$ has no coupled term between $\dot{\boldsymbol{\xi}}$ and $\dot{\boldsymbol{\eta}}$, the equation 9 can be separated into dynamics equation of $\boldsymbol{\eta}$ and $\boldsymbol{\xi}$. 
- $\boldsymbol{\xi}$ dynamic equation:

$$
\frac{d}{d t}\left(\frac{\partial L}{\partial \dot{\boldsymbol{\xi}}}\right)-\frac{\partial L}{\partial \boldsymbol{\xi}}=\boldsymbol{F} \Rightarrow m \ddot{\boldsymbol{\xi}}+\left[\begin{array}{c}
0 \\
0 \\
m g
\end{array}\right]=R \boldsymbol{F}
$$

- $\boldsymbol{\eta}$ dynamics equation is

$$
\frac{d}{d t}\left(\frac{\partial L}{\partial \dot{\boldsymbol{\eta}}}\right)-\frac{\partial L}{\partial \boldsymbol{\eta}}=\boldsymbol{\tau}
$$

thus one obtains

$$
\boldsymbol{J} \ddot{\boldsymbol{\eta}}+\dot{\boldsymbol{J}} \dot{\boldsymbol{\eta}}-\frac{1}{2} \frac{\partial}{\partial \boldsymbol{\eta}}\left(\dot{\boldsymbol{\eta}}^{T} \boldsymbol{J} \dot{\boldsymbol{\eta}}\right)=\boldsymbol{\tau}
$$

Define Coriolis-centripetal vector as

$$
\bar{V}(\boldsymbol{\eta}, \dot{\boldsymbol{\eta}})=\dot{\boldsymbol{J}} \dot{\boldsymbol{\eta}}-\frac{1}{2} \frac{\partial}{\partial \boldsymbol{\eta}}\left(\dot{\boldsymbol{\eta}}^{T} \boldsymbol{J} \dot{\boldsymbol{\eta}}\right)=\left(\dot{\boldsymbol{J}}-\frac{1}{2} \frac{\partial}{\partial \boldsymbol{\eta}}\left(\dot{\boldsymbol{\eta}}^{T} \boldsymbol{J}\right)\right) \dot{\boldsymbol{\eta}}=C(\boldsymbol{\eta}, \dot{\boldsymbol{\eta}}) \dot{\boldsymbol{\eta}}
$$

where $C(\boldsymbol{\eta}, \dot{\boldsymbol{\eta}})$ represents the Coriolis term. Finally $\boldsymbol{\eta}$ dynamic equation can be rewritten as

$$
\boldsymbol{J} \ddot{\boldsymbol{\eta}}=\boldsymbol{\tau}-C(\boldsymbol{\eta}, \dot{\boldsymbol{\eta}}) \dot{\boldsymbol{\eta}}
$$

Then the dynamic equations of quadrotor according to Euler-Lagrange approach are

$$
\begin{aligned}
& m \ddot{\boldsymbol{\xi}}+\left[\begin{array}{c}
0 \\
0 \\
m g
\end{array}\right]=R \boldsymbol{F} \\
& \ddot{\boldsymbol{\eta}}=\overline{\boldsymbol{\tau}}
\end{aligned}
$$

where $\overline{\boldsymbol{\tau}}=\boldsymbol{J}^{-1}(\boldsymbol{\tau}-C(\boldsymbol{\eta}, \dot{\boldsymbol{\eta}}) \dot{\boldsymbol{\eta}})=\left[\bar{\tau}_{\phi}, \bar{\tau}_{\theta}, \bar{\tau}_{\psi}\right]^{T}$ Finally the dynamic equation of quadrotor system is

$$
\begin{aligned}
\ddot{x} & =\frac{u_{1}}{m}(\cos \phi \sin \theta \cos \psi+\sin \phi \sin \psi) \\
\ddot{y} & =\frac{u_{1}}{m}(\cos \phi \sin \theta \sin \psi-\sin \phi \cos \psi) \\
\ddot{z} & =\frac{u_{1}}{m} \cos \phi \cos \theta-g \\
\ddot{\phi} & =\bar{\tau}_{\phi} \\
\ddot{\theta} & =\bar{\tau}_{\theta} \\
\ddot{\psi} & =\bar{\tau}_{\psi}
\end{aligned}
$$

Please notice that in the slow motion case, the Coriolis term could be relative smaller, and is ignored in the controller design. In quadrotor model $(17)$, there are four independent inputs and six degree of freedoms $(x, y, z, \phi, \theta, \psi)$. The system of inputs numbers less than degree of freedoms is called under-actuated system.

The objective in this research is to design a nonlinear controller to stabilize the quadrotor with time constraints

$$
\lim _{t \rightarrow \mathfrak{T}\left(\sigma_{0}\right)} \sigma(t)=0, \quad \mathfrak{T}\left(x_{0}\right) \leq \mathfrak{T}_{\max }
$$

and the state constraints:

$$
\begin{aligned}
\sigma_{1}^{2}+\sigma_{2}^{2} & \leq \epsilon_{1,2}^{2}, \\
\sigma_{3}^{2}+\sigma_{4}^{2} & \leq \epsilon_{3,4}^{2}, \\
\left|\sigma_{i}\right| & \leq \epsilon_{i}, \quad i=5, \ldots, 12
\end{aligned}
$$

where

$$
\sigma=(x, y, \dot{x}, \dot{y}, \phi, \theta, z, \psi, \dot{\phi}, \dot{\theta}, \dot{z}, \dot{\psi})^{T}
$$

is the system's state variables, $\mathfrak{T}$ is system's settling time function, $\mathfrak{T}_{\max }$ is the time constraint representing the maximum time of converging to origin, $\sigma_{0}$ denotes the initial state that fulfills the space constraints and the positive constants

$$
\begin{gathered}
0<\epsilon_{1,2}, \epsilon_{3,4}, \epsilon_{7}, \epsilon_{8}, \epsilon_{9}, \epsilon_{10}, \epsilon_{11}, \epsilon_{12}<+\infty \\
0<\epsilon_{5}, \epsilon_{6}<\frac{\pi}{2} \text { such that } \cos \left(\epsilon_{5}\right) \cos \left(\epsilon_{6}\right) \geq \frac{1}{2}
\end{gathered}
$$

which defines the state constraints. 


\section{System Transformation and Convex Embedding}

Denote

$$
\bar{u}=\left[\bar{\tau}_{\phi}, \bar{\tau}_{\theta}, \frac{u_{1}}{m} \cos \phi \cos \theta-g, \bar{\tau}_{\psi}\right]^{T}
$$

then quadrotor system 17 can be rewritten as the following form

$$
\dot{\sigma}=\bar{A} \sigma+B(\bar{u}+d)
$$

where $d \in \mathbb{R}^{4}$ is a constant exogenous perturbation to approximated unknown disturbance and

$$
\begin{aligned}
& \bar{A}=\bar{A}\left(\phi, \theta, \psi, u_{1}\right)=\left[\begin{array}{cccccc}
0 & I & 0 & 0 & 0 & 0 \\
0 & 0 & R E & 0 & 0 & 0 \\
0 & 0 & 0 & 0 & I & 0 \\
0 & 0 & 0 & 0 & 0 & I \\
0 & 0 & 0 & 0 & 0 & 0 \\
0 & 0 & 0 & 0 & 0 & 0
\end{array}\right], \quad E=E\left(\theta, \phi, u_{1}\right):=\left(\begin{array}{cc}
\frac{\sin \phi u_{1}}{\phi m} & 0 \\
0 & \frac{\sin \theta \cos \phi u_{1}}{\theta m}
\end{array}\right) \\
& \left.R=R(\psi):=\left(\begin{array}{cc}
\sin \psi & \cos \psi \\
-\cos \psi & \sin \psi
\end{array}\right), I=\left(\begin{array}{ll}
1 & 0 \\
0 & 1
\end{array}\right), B=\left[\begin{array}{cc}
0 & 0 \\
0 & 0 \\
0 & 0 \\
0 & 0 \\
\frac{1}{I_{x x}} & 0 \\
0 & \frac{1}{I_{y y}}
\end{array}\right] \begin{array}{cc}
0 \\
0 \\
0 \\
0
\end{array}\right]
\end{aligned}
$$

One way of simplifying the system 22 is by introducing a new variable $\zeta=T \sigma$, where $T$ in function of $\psi$ is one orthogonal matrix.

$$
T=T(\psi):=\left[\begin{array}{cccccc}
R^{-1} & 0 & 0 & 0 & 0 & 0 \\
0 & R^{-1} & 0 & 0 & 0 & 0 \\
0 & 0 & I & 0 & 0 & 0 \\
0 & 0 & 0 & I & 0 & 0 \\
0 & 0 & 0 & 0 & I & 0 \\
0 & 0 & 0 & 0 & 0 & I
\end{array}\right]
$$

Thus the studied system can be rewritten as

$$
\dot{\zeta}=(A+D) \zeta+B(\bar{u}+d), \quad \zeta(0)=\zeta_{0}:=T(\psi(0)) \sigma_{0}
$$

where

$$
A=\left[\begin{array}{cccccc}
0 & I & 0 & 0 & 0 & 0 \\
0 & 0 & E & 0 & 0 & 0 \\
0 & 0 & 0 & 0 & I & 0 \\
0 & 0 & 0 & 0 & 0 & I \\
0 & 0 & 0 & 0 & 0 & 0 \\
0 & 0 & 0 & 0 & 0 & 0
\end{array}\right], \quad D=D(\dot{\psi}):=\dot{T} T^{-1}
$$

Let $e_{i}, i=1,2, \ldots, 12$ be the identity vector in $\mathbb{R}^{12}$.

Lemma 1 Let $\Delta \in[0,1]$, the vector

$$
\epsilon=\left(\epsilon_{1,2}, \epsilon_{3,4}, \epsilon_{5}, \epsilon_{6}, \epsilon_{7}, \epsilon_{8}, \epsilon_{9}, \epsilon_{10}, \epsilon_{11}, \epsilon_{12}\right) \in \mathbb{R}_{+}^{10}
$$

satisfies 20] and

$$
A_{i}=\left[\begin{array}{cccccc}
G_{i} & I & 0 & 0 & 0 & 0 \\
0 & G_{i} & E_{i} & 0 & 0 & 0 \\
0 & 0 & 0 & 0 & I & 0 \\
0 & 0 & 0 & 0 & 0 & I \\
0 & 0 & 0 & 0 & 0 & 0 \\
0 & 0 & 0 & 0 & 0 & 0
\end{array}\right], \quad 1 \leq i \leq 8
$$

where

$$
\begin{gathered}
G_{1}=G_{3}=G_{5}=G_{7}=\epsilon_{12}\left(\begin{array}{cc}
0 & 1 \\
-1 & 0
\end{array}\right), G_{2}=G_{4}=G_{6}=G_{8}=-\epsilon_{12}\left(\begin{array}{cc}
0 & 1 \\
-1 & 0
\end{array}\right) \\
E_{1}=E_{2}=g(1+\Delta)\left(\begin{array}{cc}
1 & 0 \\
0 & 1
\end{array}\right), \quad E_{3}=E_{4}=g\left(\begin{array}{cc}
(1+\Delta) & 0 \\
0 & \frac{(1-\Delta) \sin \left(\epsilon_{6}\right) \cos \left(\epsilon_{5}\right)}{\epsilon_{6}}
\end{array}\right) \\
E_{5}=E_{6}=g\left(\begin{array}{cc}
\frac{(1-\Delta) \sin \left(\epsilon_{5}\right)}{\epsilon_{5}} & 0 \\
0 & (1+\Delta)
\end{array}\right), E_{7}=E_{8}=g(1-\Delta)\left(\begin{array}{cc}
\frac{\sin \left(\epsilon_{5}\right)}{\epsilon_{5}} & 0 \\
0 & \frac{\sin \left(\epsilon_{6}\right) \cos \left(\epsilon_{5}\right)}{\epsilon_{6}}
\end{array}\right)
\end{gathered}
$$

Then, for any $\sigma \in \mathbb{R}^{12}$ satisfying $\left[19\right.$, for any $\lambda \in[0,1]$ and for any $u_{1} \in m g[1-\Delta, 1+\Delta]$, there exist $\alpha_{i} \geq 0$ such that

$$
\sum_{i=1}^{8} \alpha_{i}=1 \quad \text { and } \quad \sum_{i=1}^{8} \alpha_{i} A_{i}=A+\lambda D
$$


Proof: If $\sigma$ satisfies $[19]$ and $u_{1} \in m g[1-\Delta, 1+\Delta]$ then, obviously, there exist $\mu_{j} \geq 0$ such that

$$
E=\mu_{1} E_{1}+\mu_{2} E_{3}+\mu_{3} E_{5}+\mu_{4} E_{7}, \quad \sum_{j=1}^{4} \mu_{j}=1 .
$$

On the other hand, since

$$
-R^{2} \dot{R} R=\dot{\psi}\left(\begin{array}{cc}
0 & 1 \\
-1 & 0
\end{array}\right)
$$

then for any $\lambda \in[0,1]$, there exist $\delta_{1}, \delta_{2} \geq 0$ such that

$$
-\lambda R^{2} \dot{R} R=\delta_{1} G_{1}+\delta_{2} G_{2}, \quad \delta_{1}+\delta_{2}=1 .
$$

Hence the simple calculation shows that

$$
A+\lambda D=A+\lambda \dot{T} T^{-1}=\sum_{i=1}^{8} \alpha_{i} A_{i}
$$

with $\alpha_{1}=\mu_{1} \delta_{1}, \alpha_{2}=\mu_{1} \delta_{2}, \alpha_{3}=\mu_{2} \delta_{1}, \alpha_{4}=\mu_{2} \delta_{2}, \alpha_{5}=\mu_{3} \delta_{1}, \alpha_{6}=\mu_{3} \delta_{2}, \alpha_{7}=\mu_{4} \delta_{1}, \alpha_{8}=\mu_{4} \delta_{2}$. Obviously, we have $\alpha_{i} \geq 0$ and $\sum_{i=1}^{8} \alpha_{i}=1 . \quad \square$ The latter lemma provides the possibility to apply the so-called convex embedding method( [4]) for implicit PD controller design.

\section{Implicit PID Controller Design with Time and state Constraint}

Using implicit Lyapunov function method to design system controller is studies in [33, let us introduce the following implicit Lyapunov function:

$$
Q(V, \zeta):=\zeta^{T} D_{r}\left(V^{-1}\right) P D_{r}\left(V^{-1}\right) \zeta-1
$$

where $0<P=P^{\top} \in \mathbb{R}^{12 \times 12}, V \in \mathbb{R}_{+}, \zeta \in \mathbb{R}^{12}$, and $D_{r}(\lambda) \in \mathbb{R}^{12 \times 12}$ is a dilation matrix in function of $\mathrm{V}$.

$$
D_{r}(\lambda)=\left[\begin{array}{cccc}
\lambda^{4} I & 0 & 0 & 0 \\
0 & \lambda^{3} I & \ldots & 0 \\
0 & 0 & \lambda^{2}\left[\begin{array}{ll}
I & 0 \\
0 & I
\end{array}\right] & 0 \\
0 & 0 & 0 & \lambda\left[\begin{array}{ll}
I & 0 \\
0 & I
\end{array}\right]
\end{array}\right], \quad \lambda \in \mathbb{R}_{+}
$$

Denote

$$
H:=\left[\begin{array}{cccccc}
4 I & 0 & 0 & 0 & 0 & 0 \\
0 & 3 I & 0 & 0 & 0 & 0 \\
0 & 0 & 2 I & 0 & 0 & 0 \\
0 & 0 & 0 & 2 I & 0 & 0 \\
0 & 0 & 0 & 0 & I & 0 \\
0 & 0 & 0 & 0 & 0 & I
\end{array}\right]
$$

Theorem 2 Select parameter

$$
\Delta \in\left[\frac{1}{\cos \left(\epsilon_{5}\right) \cos \left(\epsilon_{6}\right)}-1,1\right]
$$

and variable

$$
(X, Y, \gamma) \in \mathbb{R}^{12 \times 12} \times \mathbb{R}^{4 \times 12} \times \mathbb{R}_{+}
$$

satisfy the system of LMIs

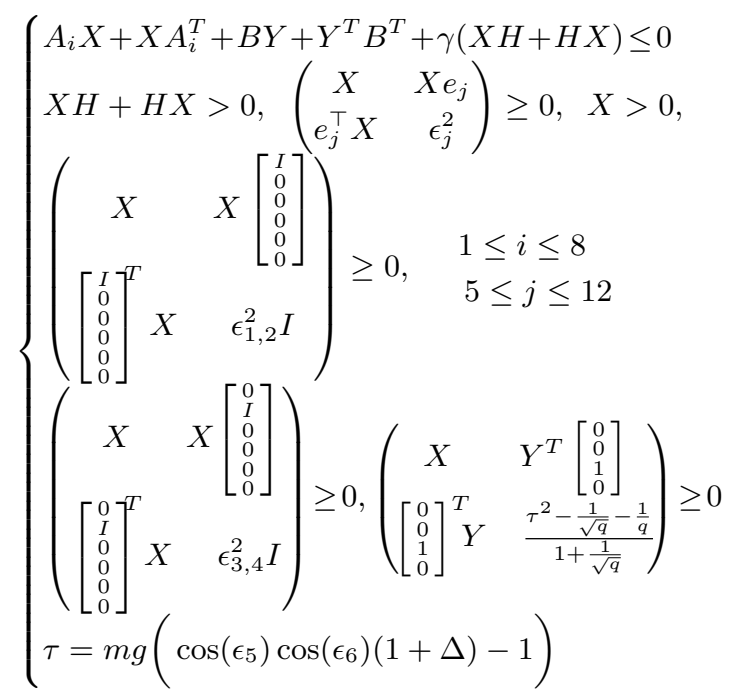


and the controller with the following form

$$
\bar{u}=K D_{r}\left(V^{-1}\right) \zeta+\int_{0}^{t} K_{I} D_{r}\left(V^{-1}\right) \zeta(s) d s
$$

where

$$
\begin{gathered}
K=Y X^{-1}, \quad K_{I}=-\frac{P_{I}^{-1} B^{T} P}{\zeta^{T} D_{r}\left(V^{-1}\right)(P H+H P) D_{r}\left(V^{-1}\right) \zeta} \\
V \in \mathbb{R}_{+}: \zeta^{T} D_{r}\left(V^{-1}\right) P D_{r}\left(V^{-1}\right) \zeta=1,
\end{gathered}
$$

then for any initial condition $\zeta(0)=\zeta_{0}$ satisfying

$$
\begin{gathered}
\zeta_{0}^{T} D_{r}\left(\left(1-d^{T} P_{I} d\right)^{-1}\right) P D_{r}\left(\left(1-d^{T} P_{I} d\right)^{-1}\right) \zeta_{0} \leq 1 \\
P=X^{-1}
\end{gathered}
$$

where $0<P_{I}=q I, P_{I} \in \mathbb{R}^{4 \times 4}$ such that $d^{T} P_{I} d<1$, the system 24 converges to zero in a finite time

Moreover the control $\bar{u}$ is bounded by

$$
\mathfrak{T}\left(\zeta_{0}\right) \leq \frac{V\left(\sigma_{0}\right)}{\gamma} \leq \frac{1}{\gamma}
$$

$$
\|\bar{u}\|^{2} \leq\left(1+\frac{1}{\sqrt{q}}\right) \lambda_{\max }\left(P^{-\frac{1}{2}} K^{T} K P^{-\frac{1}{2}}\right)+\frac{1}{\sqrt{q}}+\frac{1}{q}
$$

and the state constraints defined in 19 are fulfilled for all $t \geq 0$.

Proof: The system 24 with controller 30 and disturbance $d$ could be rewritten in the following form

$$
\dot{\bar{\zeta}}=\left[\begin{array}{cc}
A+D & B \\
0 & 0
\end{array}\right] \bar{\zeta}+\left[\begin{array}{ll}
B & 0 \\
0 & I
\end{array}\right] \bar{K} \bar{\zeta}
$$

where $\bar{\zeta}=\left[\zeta, \zeta_{n+1}\right]^{T}$, and suppose $\zeta_{n+1}=\int_{0}^{t} K_{I} D_{r}\left(V^{-1}\right) \zeta(s) d s+d, \bar{K}=\left[\begin{array}{cc}K D\left(V^{-1}\right) & 0 \\ K_{I} D\left(V^{-1}\right) & 0\end{array}\right]$.

Following [34, it is the time to introduce the following extended Lyapunov function:

$$
\bar{V}=V+\zeta_{n+1}^{T} P_{I} \zeta_{n+1}
$$

Since $V$ is the solution of 31 , it is easy to develop the following result under the initial condition 32 :

$$
\zeta_{0}^{T} D_{r}\left(\left(1-d^{T} P_{I} d\right)^{-1}\right) P D_{r}\left(\left(1-d^{T} P_{I} d\right)^{-1}\right) \zeta_{0} \leq \zeta_{0}^{T} D_{r}\left(V(0)^{-1}\right) P D_{r}\left(V(0)^{-1}\right) \zeta_{0}
$$

which is equivalent to

$$
\begin{gathered}
V(0) \leq 1-d^{T} P_{I} d, \quad d=\zeta_{n+1}(0) \\
\bar{V}(0)=V(0)+d^{T} P_{I} d \leq 1
\end{gathered}
$$

In [33, it was shown that the implicit Lyapunov function of the form 27] satisfies the conditions C1)-C3) of Theorem 1 Since

$$
\frac{\partial Q(V, \zeta)}{\partial V}=-V^{-1} \zeta^{T} D_{r}\left(V^{-1}\right)(P H+H P) D_{r}\left(V^{-1}\right) \zeta
$$

$P=X^{-1}$ and $X H+H X>0$, then $\frac{\partial Q}{\partial V}<0$ for $\forall V \in \mathbb{R}_{+}$and $\zeta \in \mathbb{R}^{12} \backslash\{0\}$. So the condition C4) of Theorem 1 also holds.

Since $D_{r}\left(V^{-1}\right) A D_{r}^{-1}\left(V^{-1}\right)=V^{-1} A$ and $D_{r}\left(V^{-1}\right) B \bar{u}=V^{-1} B K D_{r}\left(V^{-1}\right) \zeta$, then we have

$$
\begin{aligned}
& \frac{\partial Q(V, \zeta)}{\partial \zeta}(A \zeta+B \bar{u}+D \zeta)=2 \frac{\zeta^{T} D_{r}\left(V^{-1}\right)(P A+P B K+V P D) D_{r}\left(V^{-1}\right) \zeta}{V} \\
& =2 \sum_{i=1}^{8} \alpha_{i} \frac{\zeta^{T} D_{r}\left(V^{-1}\right)\left(P A_{i}+P B K\right) D_{r}\left(V^{-1}\right) \zeta+\zeta^{T} D_{r}\left(V^{-1}\right) P B \zeta_{n+1}}{V}
\end{aligned}
$$

with $\alpha_{i} \geq 0, \quad \sum_{i=1}^{8} \alpha_{i}=1$, where Lemma 1 is utilized on the last step. Therefore, we can obtain the following inequalities:

$$
\begin{aligned}
\dot{V} & =-\left(\frac{\partial Q(V, \zeta)}{\partial V}\right)^{-1} \frac{\partial Q(V, \zeta)}{\partial \zeta}((A+D) \zeta+B \bar{u}) \\
& \leq 2 \sum_{i=1}^{8} \alpha_{i} \frac{\zeta^{T} D_{r}\left(V^{-1}\right)\left(P A_{i}+P B K\right) D_{r}\left(V^{-1}\right) \zeta+\zeta^{T} D_{r}\left(V^{-1}\right) P B \zeta_{n+1}}{\zeta^{T} D_{r}\left(V^{-1}\right)(P H+H P) D_{r}\left(V^{-1}\right) \zeta} \\
& \leq-\gamma+2 \frac{\zeta^{T} D_{r}\left(V^{-1}\right) P B \zeta_{n+1}}{\zeta^{T} D_{r}\left(V^{-1}\right)(P H+H P) D_{r}\left(V^{-1}\right) \zeta}
\end{aligned}
$$


and

$$
\begin{aligned}
\dot{\bar{V}} & =\dot{V}+2 \dot{\zeta}_{n+1}^{T} P_{I} \zeta_{n+1} \\
& \leq-\gamma+2 \frac{\zeta^{T} D_{r}\left(V^{-1}\right) P B \zeta_{n+1}}{\zeta^{T} D_{r}\left(V^{-1}\right)(P H+H P) D_{r}\left(V^{-1}\right) \zeta}+2 \zeta^{T} D_{r}\left(V^{-1}\right) K_{i}^{T} P_{I} \zeta_{n+1} \\
& \leq-\gamma<0
\end{aligned}
$$

According to 36 and $(37)$, we have

$$
\bar{V}(t) \leq 1 \Rightarrow V(t) \leq 1
$$

and the system 34 converges to 0 in finite time

$$
\mathfrak{T}\left(\zeta_{0}\right) \leq \frac{1}{\gamma}
$$

provided that the phase constraints are fulfilled, $V \leq 1$ and $u_{1} \in m g[1-\Delta, 1+\Delta]$.

To complete the proof, let us show that the phase constrains and the inclusion $u_{1} \in m g[1-\Delta, 1+\Delta]$ hold if $V \leq 1$ (or, equivalently, $\zeta^{\top} P \zeta \leq 1$ ). Indeed, the required phase constraints for $j=5, \ldots, 12$ comes from

$$
\begin{aligned}
{\left[\begin{array}{cc}
\epsilon_{j}^{2} X & X e_{j} \\
e_{j}^{\top} X & 1
\end{array}\right] \geq 0 } & \Leftrightarrow X e_{j} e_{j}^{\top} X \leq \epsilon_{j}^{2} X \\
& \Leftrightarrow e_{j} e_{j}^{\top} \leq \epsilon_{j}^{2} P \\
& \Leftrightarrow \zeta_{j}^{2}=\zeta^{T} e_{j} e_{j}^{\top} \zeta \leq \epsilon_{j}^{2} \zeta^{T} P \zeta \leq \epsilon_{j}^{2}
\end{aligned}
$$

The constraints for $\sigma_{1}, \sigma_{2}, \sigma_{3}$ and $\sigma_{4}$ can be checked similarly by taking into account that

$$
\left[\begin{array}{l}
\sigma_{1} \\
\sigma_{2}
\end{array}\right]^{\top}\left[\begin{array}{l}
\sigma_{1} \\
\sigma_{2}
\end{array}\right]=\left[\begin{array}{l}
\sigma_{1} \\
\sigma_{2}
\end{array}\right]^{\top} R^{\top} R\left[\begin{array}{l}
\sigma_{1} \\
\sigma_{2}
\end{array}\right]=\left[\begin{array}{l}
\zeta_{1} \\
\zeta_{2}
\end{array}\right]^{\top}\left[\begin{array}{l}
\zeta_{1} \\
\zeta_{2}
\end{array}\right]
$$

Since $\zeta^{T} D_{r}\left(V^{-1}\right) P D_{r}\left(V^{-1}\right) \zeta=1$, by using Young's inequality, the norm square of controller (34) can be estimated as follows

$$
\begin{aligned}
\|\bar{u}\|^{2} & \leq\left(1+\sqrt{q}^{-1}\right) \zeta^{T} D_{r}\left(V^{-1}\right) K^{T} K D_{r}\left(V^{-1}\right) \zeta+\left(1+\sqrt{q}^{-1}\right) \zeta_{n+1}^{T} \zeta_{n+1} \\
& =\left(1+\sqrt{q}^{-1}\right) \zeta^{T} D_{r}\left(V^{-1}\right) K^{T} K D_{r}\left(V^{-1}\right) \zeta+\frac{\left(1+\sqrt{q}^{-1}\right) \zeta_{n+1}^{T} \zeta_{n+1} q}{q}
\end{aligned}
$$

Since $\bar{V}<1$, we have $\zeta_{n+1}^{T} \zeta_{n+1} q<1$ then

$$
\|\bar{u}\|^{2} \leq\left(1+\frac{1}{\sqrt{q}}\right) \lambda_{\max }\left(P^{-\frac{1}{2}} K^{T} K P^{-\frac{1}{2}}\right)+\frac{1}{\sqrt{q}}+\frac{1}{q}
$$

Similarly we derive

$$
\|\bar{u}\|_{1}^{2} \leq\left(1+\frac{1}{\sqrt{q}}\right) \lambda_{\max }\left(P^{-\frac{1}{2}} K^{T}\left[\begin{array}{l}
0 \\
0 \\
1 \\
0
\end{array}\right]\left[\begin{array}{l}
0 \\
0 \\
1 \\
0
\end{array}\right]^{T} K P^{-\frac{1}{2}}\right)+\frac{1}{\sqrt{q}}+\frac{1}{q} \leq \tau^{2}
$$

which is equivalent to the last inequality of 29 .

$\square$ Please note that the state constraints can be added or removed depending on the controller design requirement, then the corresponding LMIs need to do the same processing in in 29. $\gamma$ is a parameter for tuning the settling time, which can be minimized by finding the maximum feasible value of $\gamma$.

$$
\gamma \rightarrow \gamma_{\max }
$$

subject to 29 .

Remark: When the disturbance $d$ is zero, then we can select $K_{I}=0$ and the controller becomes the PD controller proposed in [36].

\section{Robustness Analysis}

In the practice, the quadrotor system generally not only has some static errors, but also some unknown dynamic perturbations. The following Corollary studies the quadrotor model with unknown dynamic perturbations. The perturbation can be modeled by a set-valued or discontinuous function provided that Filippov solution exists in the closed-loop system [4]. 
Corollary 1 Let the conditions of Theorem 2 hold and $F: \mathbb{R} \times \mathbb{R}^{n} \rightrightarrows \mathbb{R}^{n}$ is a compact-valued, convex-valued and upper-semi continuous satisfying the following inequality

$$
\frac{\sup _{y \in F(t, \zeta)\left\|D_{r}\left(V^{-1}\right) y\right\|_{p}}}{\zeta^{\top} D_{r}^{\top}\left(V^{-1}\right)(P H) D_{r}\left(V^{-1}\right) \zeta} \leq \kappa V^{-1}, \quad \forall x \in \mathbb{R}^{n}, \forall t \geq 0
$$

where $\kappa>0$ and additionally

$$
A_{i} X+X A_{i}^{\top}+B Y+Y^{\top} B^{\top}+(\gamma+\kappa)(X H+H X) \leq 0, \quad 1 \leq i \leq 8
$$

then the controller (30) stabilizes the system

$$
\dot{\zeta} \in(A+D) \zeta+B(\bar{u}+d)+F(t, \zeta), \quad t>0
$$

in finite-time.

Proof: The existence of solutions of the closed-loop system follows from the Filippov theory [44. Follow the similar steps in the proof of Theorem 2 one can derive that

$$
\begin{aligned}
\dot{V} & \leq 2 \sum_{i=1}^{8} \alpha_{i} \frac{\zeta^{T} D_{r}\left(V^{-1}\right)\left(P A_{i}+P B K\right) D_{r}\left(V^{-1}\right) \zeta+\zeta^{T} D_{r}\left(V^{-1}\right) P B \zeta_{n+1}}{\zeta^{T} D_{r}\left(V^{-1}\right)(P H+H P) D_{r}\left(V^{-1}\right) \zeta} \\
& +2 V \sup _{y \in F(t, \zeta)} \frac{\zeta^{T} D_{r}\left(V^{-1}\right) P D_{r}\left(V^{-1}\right) y}{\zeta^{T} D_{r}\left(V^{-1}\right)(P H+H P) D_{r}\left(V^{-1}\right) \zeta}
\end{aligned}
$$

Using the Cauchy-Schwarz inequality and $\zeta^{\top} D_{r}^{\top}\left(V^{-1}\right) P D_{r}\left(V^{-1}\right) \zeta=1$, we have

$$
\begin{aligned}
\zeta^{T} D_{r}^{\top}\left(V^{-1}\right) P D_{r}\left(V^{-1}\right) y & \leq\left\|D_{r}\left(V^{-1}\right) \zeta\right\|_{p} \| D_{r}\left(V^{-1} y \|_{p}\right. \\
& \leq\left\|D_{r}\left(V^{-1}\right) y\right\|_{p}
\end{aligned}
$$

Then apply the LMI 41, , one can derive

$$
\begin{aligned}
\dot{V} & =-(\gamma+\kappa)+2 \frac{\zeta^{T} D_{r}\left(V^{-1}\right) P B \zeta_{n+1}}{\zeta^{T} D_{r}\left(V^{-1}\right)(P H+H P) D_{r}\left(V^{-1}\right) \zeta}+\kappa \\
& =-\gamma+2 \frac{\zeta^{T} D_{r}\left(V^{-1}\right) P B \zeta_{n+1}}{\zeta^{T} D_{r}\left(V^{-1}\right)(P H+H P) D_{r}\left(V^{-1}\right) \zeta}
\end{aligned}
$$

and repeat the proof in Theorem 2 , we have

$$
\begin{aligned}
\dot{\bar{V}} & =\dot{V}+2 \dot{\zeta}_{n+1}^{T} P_{I} \zeta_{n+1} \\
& \leq-\gamma<0
\end{aligned}
$$

$\square$ Notice that the above case represents the disturbance in function of $\zeta$ that will vanish as $\zeta$ converges to zero.

In the particular case where $D_{r}\left(V^{-1}\right) B=V^{-1} B$ and $F(t, \zeta)=B \Lambda(t, \zeta)$ with $\Lambda: \mathbb{R} \times \mathbb{R}^{n} \rightrightarrows \mathbb{R}^{m}$ which means the disturbance is matched, then the inequality 40 becomes

$$
\frac{\sup _{\nu \in \Lambda(t, \zeta)\|B \nu\|_{p}}}{\zeta^{\top} D_{r}^{\top}\left(V^{-1}\right) P H D_{r}\left(V^{-1}\right) \zeta} \leq \kappa, \quad \forall x \in \mathbb{R}^{n}, \forall t \geq 0
$$

Since $\left\|D_{r}\left(V^{-1}\right) \zeta\right\|_{p}=1$ and $P H+H P>0$ then there is

$$
c_{\text {min }}:=\inf \zeta^{\top} D_{r}^{\top}\left(V^{-1}\right) P H D_{r}\left(V^{-1}\right) \zeta>0
$$

which means if the additional LMI 41) holds, then the homogeneous controller (30) can reject bounded perturbation $B \Lambda$ with magnitude $\kappa c_{\min }>0$.

If there is a mismatched bounded disturbance for system [42, the Theorem 3.3 in 45] has proved that if the system is locally homogeneous and globally asymptotically stable (system 42 satisfies this condition with homogeneous controller (30), then the it is locally ISS with respect to homogeneously involved perturbation. 
Table 1: Quadrotor Parameters

\begin{tabular}{|c|c|c|c|}
\hline Parameter & Description & Value & Units \\
\hline \hline $\mathrm{g}$ & Gravity & 9.8 & $\mathrm{~m} / \mathrm{s}^{2}$ \\
$\mathrm{~m}$ & Mass & 1.07 & $\mathrm{~kg}$ \\
$L_{\text {roll }}$ & Roll motor distance & 0.2136 & $\mathrm{~m}$ \\
$L_{\text {pitch }}$ & Pitch motor distance & 0.1758 & $\mathrm{~m}$ \\
$I_{x x}$ & Roll Inertia & $6.85 \times 10^{-3}$ & $\mathrm{kgm}^{2}$ \\
$I_{y y}$ & Pitch Inertia & $6.62 \times 10^{-3}$ & $\mathrm{kgm}^{2}$ \\
$I_{z z}$ & Yaw Inertia & $1.29 \times 10^{-2}$ & $\mathrm{kgm}^{2}$ \\
$k$ & Thrust Coefficient & $1.93 \times 10^{-8}$ & $\frac{\mathrm{N}}{R M^{2}}$ \\
$c$ & Drag Coefficient & $0.26 \times 10^{-9}$ & $\frac{\mathrm{Nm}^{2}}{R P M^{2}}$ \\
\hline
\end{tabular}

\section{SIMULATION RESULTS}

In this section, the simulation results are based the quadrotor parameters of table 1 Suppose that the quadrotor needs to be stabilized under the following constraints

$$
|\dot{\psi}| \leq 0.9, \quad|\phi| \leq \frac{\pi}{6}, \quad|\theta| \leq \frac{\pi}{6}
$$

Solving LMIs 29) gives the following gain matrix

$$
K=\left[\begin{array}{cccccccccccc}
-65.00 & 0 & -76.84 & 0 & -199.95 & 0 & 0 & 0 & -1.24 & 0 & 0 & 0 \\
0 & -110.09 & 0 & -134.17 & 0 & -332.58 & 0 & 0 & 0 & -1.91 & 0 & 0 \\
0 & 0 & 0 & 0 & 0 & 0 & -0.30 & 0 & 0 & 0 & -0.83 & 0 \\
0 & 0 & 0 & 0 & 0 & 0 & 0 & -20.56 & 0 & 0 & 0 & -35.96
\end{array}\right]
$$

The initial condition here is

$$
\sigma_{0}=[0.23 ;-0.22 ; 0 ; 0 ; 0 ; 0 ; 0.4 ; 0.15 ; 0 ; 0 ; 0 ; 0]
$$

which makes $\sigma_{0}^{T} P \sigma_{0}=0.5766<1, d=\left[\begin{array}{llll}0.1 & 0.1 & 0.65 & 0.1\end{array}\right]^{T}$ and $\gamma=0.21$.

Fig. 2 and Fig. 3 show that the trajectory of position and attitude converge to origin in finite time under the implicit PID controller, and all states converge to zero less than $\frac{1}{\gamma}=4.77 \mathrm{~s}$. It is clear to see that the position and attitude state converge simultaneously to the origin, since the controller design is based on the full states of system. The constraints $|\dot{\psi}|<0.9,|\theta|<\frac{\pi}{6},|\phi|<\frac{\pi}{6}$ are satisfied and confirmed by Fig 3 and Fig 4 . Fig 5 shows that the $\log \|v\|$ with $v=[x, y, \phi, \theta, z, \psi]$ decreases in a nonlinear manner, which is the convergence property of finite time controller.

The simulation results prove the nonlinear implicit PID controller can stabilize the quadrotor to the origin under state and time constrains and is robust even if there are some initial constant errors.

Fig. 6 shows that if the quadrotor system has a static error $d=[0.1 ; 0.1 ; 0.65 ; 0.1]$, the position can not converge to the desired position without integrator term (which is the implicit PD controller proposed in 36]).

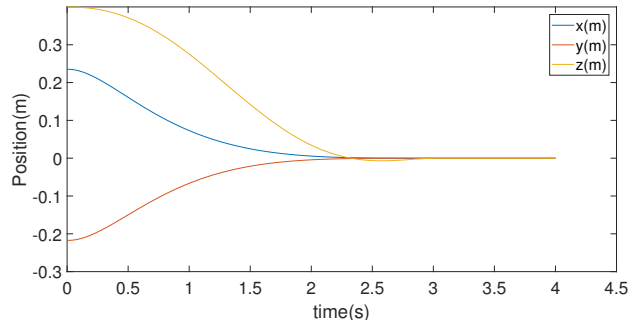

Figure 2: Position: implicit PID under $d$

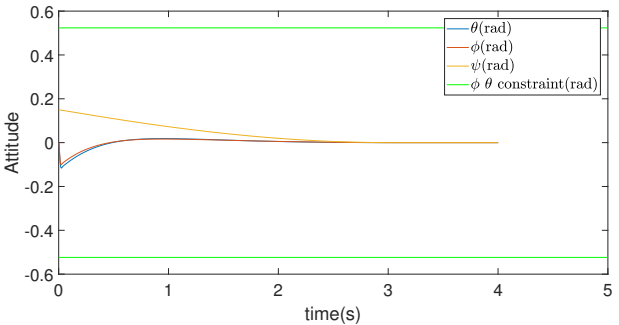

Figure 3: Attitude: implicit PID under $d$

In the above simulation, the disturbance is setted to be constant and matched with input, the following simulation results characterize the system stability under mismatched disturbance and mismatched disturbance vanishing with system state.

The system parameters (controller gain,constraints and $\gamma$ )are the same to the previous simulations. In the case of mismatched disturbance, assume that $f_{1} \in F(t, \zeta)$

$$
f_{1}=[0.05 ; 0.05 ; 0.01 ; 0.01 ; 0.01 ; 0.01 ; 0 ; 0 ; 0 ; 0 ; 0.01 ; 0.01] * \sin (t)
$$




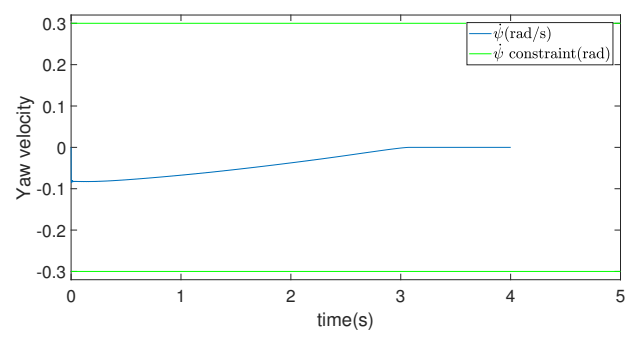

Figure 4: $\dot{\psi}$ : implicit PID under d

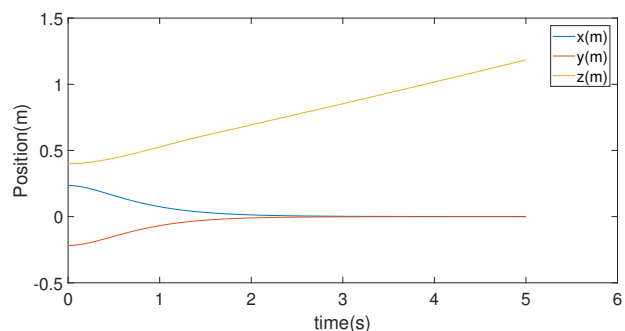

Figure 6: Position: implicit PD under $d$

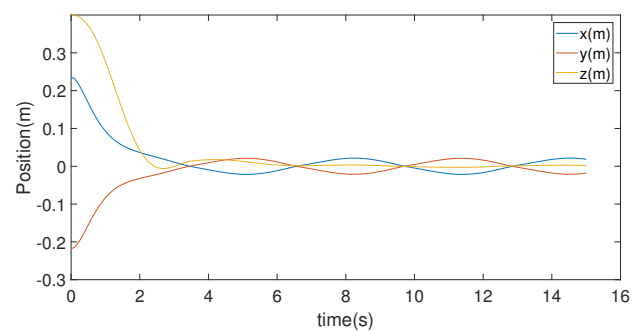

Figure 8: Position: implicit PID under $d$ and $f_{1}$

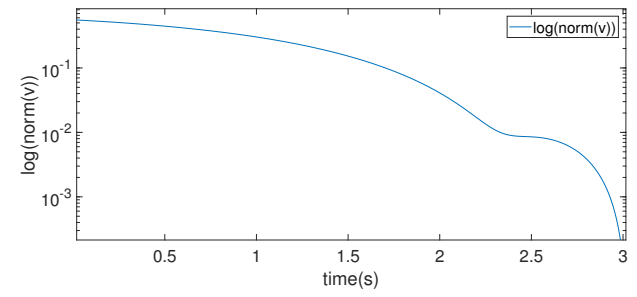

Figure 5: $\log (\|v\|)$ : implicit PID under $d$

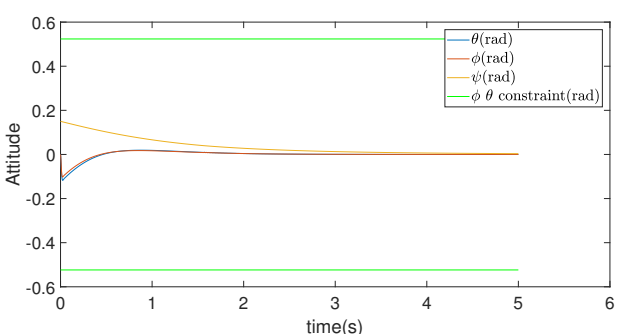

Figure 7: Attitude: implicit PD under $d$

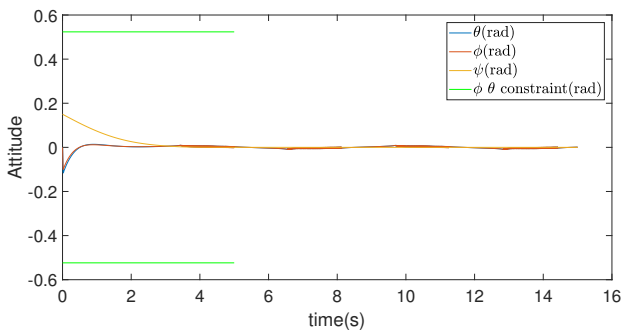

Figure 9: Attitude: implicit PID under $d$ and $f_{1}$ 
Fig. 8 and Fig. 9 depict the trajectory of position and attitude of quadrotor under disturbance $d$ and $f_{1}$. It is clear to see that the system states converge to certain invariant set, less than 3 second. The error of upper/lower bound for $\mathrm{x}$ and $\mathrm{y}$ is $0.0215 /-0.0215$. In the case of mismatched disturbance vanishing with $\zeta$, assume that $f_{2} \in F(t, \zeta)$

$$
f_{2}=[0.05 ; 0.05 ; 0.01 ; 0.01 ; 0.01 ; 0.01 ; 0 ; 0 ; 0 ; 0 ; 0.01 ; 0.01] * \zeta(t)
$$

Then Fig. 10 and Fig. 11 present the system stability of this case. Obviously, all the system state converges to the origin before $t=3.5 \mathrm{~s}$ as the system state $\zeta$ converges to zero.

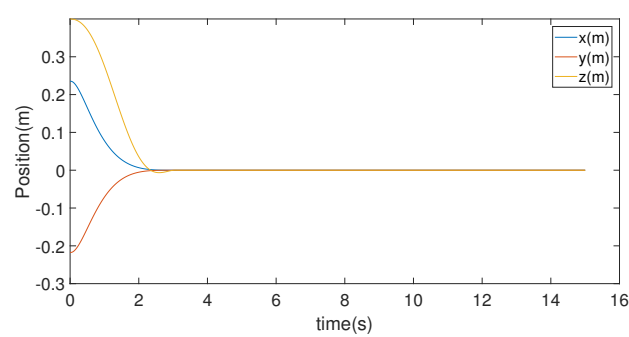

Figure 10: Position: implicit PID under $d$ and $f_{2}$

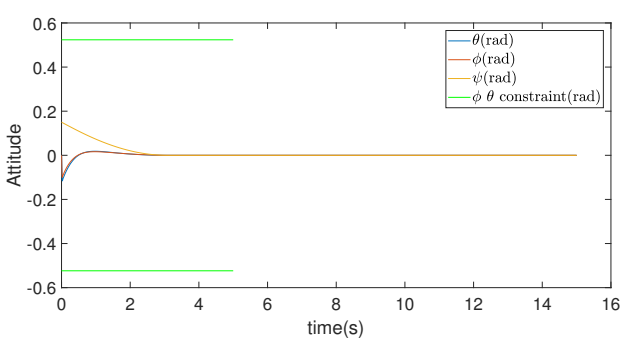

Figure 11: Attitude: implicit PID under $d$ and $f_{2}$

In order to have a more realistic control results, a first order time-delay system

$$
\epsilon \dot{\tilde{u}}=\bar{u}(t-\tau)-\tilde{u}, \quad \epsilon=10^{-3}, \tau=0.01
$$

can be added in the control loop. In this case, one important issue of implicit PID controller 30 is that as $\zeta \rightarrow 0$ with error, the corresponding gain will tend to infinity which leads to a chattering problem, see Fig 12 where the chattering $V \in(0.170,0.370)$.

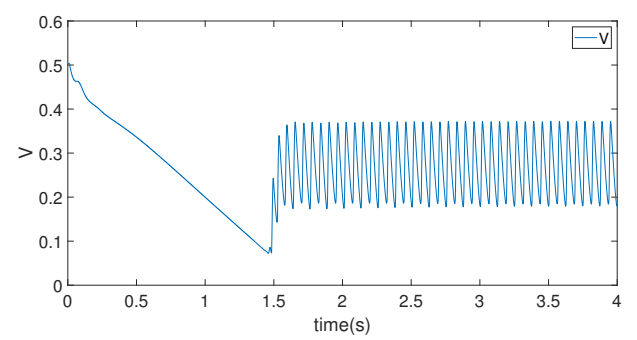

Figure 12: V: implicit PID with chattering

To avoid this infinite gain and chattering problem, a saturation function can be applied as follows

$$
\operatorname{sat}_{a, b}(V)=\left\{\begin{array}{l}
V_{b} \text { if } \quad V \geq V_{b} \\
V \quad \text { if } \quad V_{a} \leq V \leq V_{b} \\
V_{a} \text { if } \quad V \leq V_{a}
\end{array}\right.
$$

A new form implicit PID controller with saturation function can be rewritten as follows

$$
\bar{u}=K D_{r}\left(\left(\operatorname{sat}_{a, b}(V)\right)^{-1}\right) \zeta+\int_{0}^{t} K_{I} D_{r}\left(\left(\operatorname{sat}_{a, b}(V)\right)^{-1}\right) \zeta(s) d s
$$

Fig. 13-17 present one simulation result of implicit PID controller with first order time-delay and disturbance $d$, where $V_{a}=0.2, V_{b}=1$. In this case, we relax the constraints of state and only keep the time constraint. With $\gamma=0.1$, solving the LMIs 29] gives

$$
K=\left[\begin{array}{cccccccccccc}
-0.19 & 0 & -0.23 & 0 & -0.82 & 0 & 0 & 0 & -0.087 & 0 & 0 & 0 \\
0 & -0.20 & 0 & -0.25 & 0 & -0.82 & 0 & 0 & 0 & -0.085 & 0 & 0 \\
0 & 0 & 0 & 0 & 0 & 0 & -4.54 & 0 & 0 & 0 & -2.86 & 0 \\
0 & 0 & 0 & 0 & 0 & 0 & 0 & -0.05 & 0 & 0 & 0 & -0.03
\end{array}\right]
$$

All the system states are stabilized after $t=2 \mathrm{~s}$ without any chattering problem. 


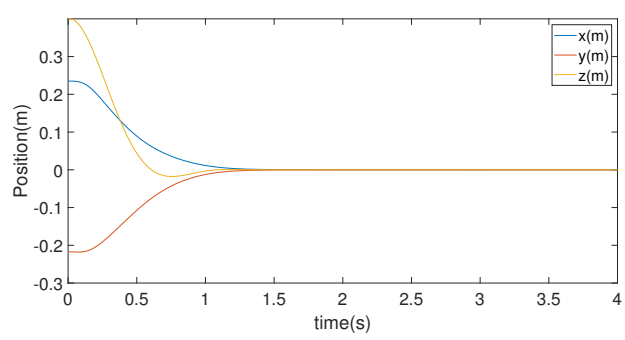

Figure 13: Position: implicit PID with $d$ and time-delay

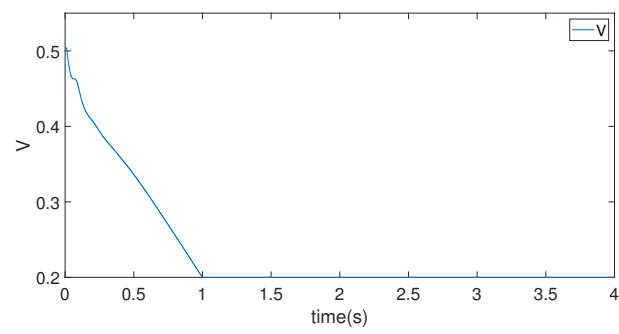

Figure 15: V: implicit PID with $d$ and time-delay

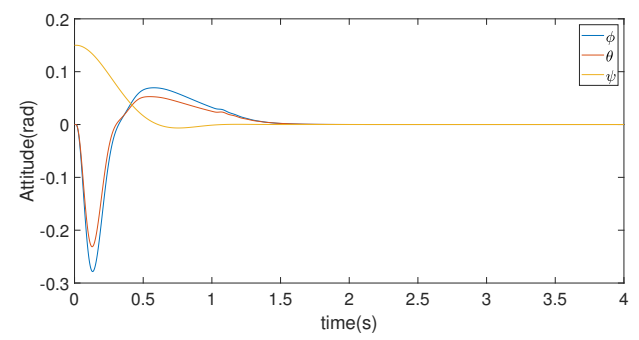

Figure 14: Attitude: implicit PID with $d$ and time-delay

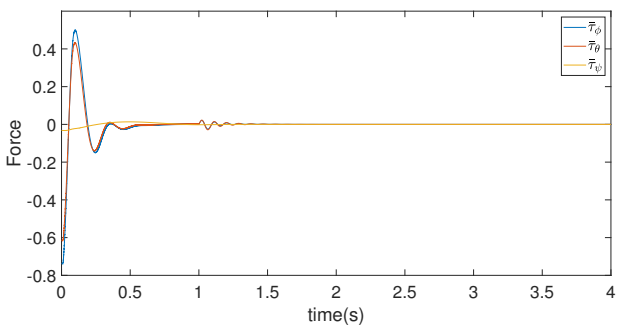

Figure 16: Inputs: implicit PID with $d$ and time-delay

\section{Conclusion}

In this paper, the problem of finite-time stabilization of quadrotor under state constraints and bounded disturbance has been studied. Convex embedding technique is utilized to construct LMIs from which the optimal feedback gains is solved. The property of stability and robustness for the proposed nonlinear implicit PID controller is verified by simulation with bounded disturbance and time-delay input, which shows its application prospects. The main limit of this research is that how the model uncertainty effects the closed-loop system stability and performance, which is one direction of the future research. Another limit is the assumption of precisely knowing all physical parameters. Hence one of our future works to design observer to identify those parameters, which will be then used in the closed-loop control.

\section{References}

[1] S. Bouabdallah, A. Noth, R. Siegwart, Pid vs lq control techniques applied to an indoor micro quadrotor, in: 2004 IEEE/RSJ International Conference on Intelligent Robots and Systems (IROS)(IEEE Cat. No. 04CH37566), Vol. 3, IEEE, 2004, pp. 2451-2456.

[2] J. Li, Y. Li, Dynamic analysis and pid control for a quadrotor, in: 2011 IEEE International Conference on Mechatronics and Automation, IEEE, 2011, pp. 573-578.

[3] R. Mahony, V. Kumar, P. Corke, Multirotor aerial vehicles: Modeling, estimation, and control of quadrotor, IEEE Robotics and Automation magazine 19 (3) (2012) 20-32.

[4] E. Reyes-Valeria, R. Enriquez-Caldera, S. Camacho-Lara, J. Guichard, Lqr control for a quadrotor using unit quaternions: Modeling and simulation, in: CONIELECOMP 2013, 23rd International Conference on Electronics, Communications and Computing, IEEE, 2013, pp. 172-178.

[5] H. Liu, G. Lu, Y. Zhong, Robust lqr attitude control of a 3-dof laboratory helicopter for aggressive maneuvers, IEEE Transactions on Industrial Electronics 60 (10) (2012) 4627-4636.

[6] A. Mokhtari, A. Benallegue, B. Daachi, Robust feedback linearization and gh/sub/spl infin//controller for a quadrotor unmanned aerial vehicle, in: 2005 IEEE/RSJ International Conference on Intelligent Robots and Systems, IEEE, 2005, pp. 1198-1203.

[7] J. Gadewadikar, F. L. Lewis, K. Subbarao, K. Peng, B. M. Chen, H-infinity static outputfeedback control for rotorcraft, Journal of Intelligent and Robotic Systems 54 (4) (2009) 629-646.

[8] D. Lee, H. J. Kim, S. Sastry, Feedback linearization vs. adaptive sliding mode control for a quadrotor helicopter, International Journal of control, Automation and systems 7 (3) (2009) 419-428. 


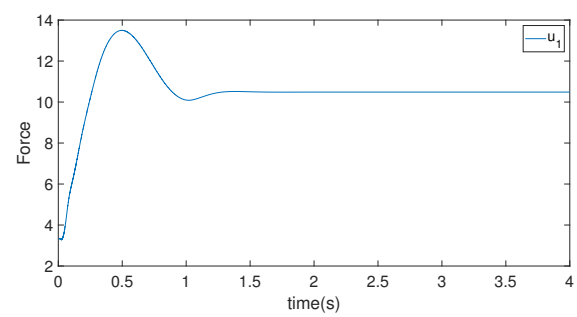

Figure 17: Thrust $u_{1}$ : implicit PID with $d$ and time-delay

[9] H. Voos, Nonlinear control of a quadrotor micro-uav using feedback-linearization, in: 2009 IEEE International Conference on Mechatronics, IEEE, 2009, pp. 1-6.

[10] G. V. Raffo, M. G. Ortega, F. R. Rubio, Mpc with nonlinear h infinite control for path tracking of a quad-rotor helicopter, IFAC Proceedings Volumes 41 (2) (2008) 8564-8569.

[11] K. Alexis, G. Nikolakopoulos, A. Tzes, Model predictive quadrotor control: attitude, altitude and position experimental studies, IET Control Theory \& Applications 6 (12) (2012) 1812-1827.

[12] M. Bangura, R. Mahony, Real-time model predictive control for quadrotors, IFAC Proceedings Volumes 47 (3) (2014) 11773-11780.

[13] S. Bouabdallah, R. Siegwart, Backstepping and sliding-mode techniques applied to an indoor micro quadrotor, in: Proceedings of the 2005 IEEE international conference on robotics and automation, IEEE, 2005, pp. 2247-2252.

[14] J. Colorado, A. Barrientos, A. Martinez, B. Lafaverges, J. Valente, Mini-quadrotor attitude control based on hybrid backstepping \& frenet-serret theory, in: 2010 IEEE International Conference on Robotics and Automation, IEEE, 2010, pp. 1617-1622.

[15] T. Madani, A. Benallegue, Backstepping control for a quadrotor helicopter, in: 2006 IEEE/RSJ International Conference on Intelligent Robots and Systems, IEEE, 2006, pp. 3255-3260.

[16] R. Xu, U. Ozguner, Sliding mode control of a quadrotor helicopter, in: Proceedings of the 45th IEEE Conference on Decision and Control, IEEE, 2006, pp. 4957-4962.

[17] L. Luque-Vega, B. Castillo-Toledo, A. G. Loukianov, Robust block second order sliding mode control for a quadrotor, Journal of the Franklin Institute 349 (2) (2012) 719-739.

[18] L. Derafa, A. Benallegue, L. Fridman, Super twisting control algorithm for the attitude tracking of a four rotors uav, Journal of the Franklin Institute 349 (2) (2012) 685-699.

[19] L. Fridman, A. Levant, et al., Higher order sliding modes, Sliding mode control in engineering 11 (2002) 53-102.

[20] E. Cruz-Zavala, J. A. Moreno, Homogeneous high order sliding mode design: a lyapunov approach, Automatica 80 (2017) 232-238.

[21] A. Polyakov, D. Efimov, W. Perruquetti, Finite-time and fixed-time stabilization: Implicit lyapunov function approach, Automatica 51 (2015) 332-340.

[22] F. Mazenc, R. Mahony, R. Lozano, Forwarding control of scale model autonomous helicopter: a lyapunov control design, in: 42nd IEEE International Conference on Decision and Control (IEEE Cat. No. 03CH37475), Vol. 4, IEEE, 2003, pp. 3960-3965.

[23] S. Li, Y. Wang, J. Tan, Adaptive and robust control of quadrotor aircrafts with input saturation, Nonlinear Dynamics 89 (1) (2017) 255-265.

[24] N. Metni, T. Hamel, A uav for bridge inspection: Visual servoing control law with orientation limits, Automation in construction 17 (1) (2007) 3-10.

[25] M. Bürger, M. Guay, A backstepping approach to multivariable robust constraint satisfaction with application to a vtol helicopter, in: Proceedings of the 48h IEEE Conference on Decision and Control (CDC) held jointly with 2009 28th Chinese Control Conference, IEEE, 2009, pp. $5239-5244$

[26] A. Rodić, G. Mester, I. Stojković, Qualitative evaluation of flight controller performances for autonomous quadrotors, in: Intelligent Systems: Models and Applications, Springer, 2013, pp. $115-134$

[27] N. Cao, A. F. Lynch, Inner-outer loop control for quadrotor uavs with input and state constraints, IEEE Transactions on Control Systems Technology 24 (5) (2016) 1797-1804. 
[28] T. Hirata, M. Kumon, Optimal path planning method with attitude constraints for quadrotor helicopters, in: Proceedings of the 2014 International Conference on Advanced Mechatronic Systems, IEEE, 2014, pp. 377-381.

[29] B. Tian, H. Lu, Z. Zuo, Q. Zong, Y. Zhang, Multivariable finite-time output feedback trajectory tracking control of quadrotor helicopters, International Journal of Robust and Nonlinear Control 28 (1) (2018) 281-295.

[30] H. Du, W. Zhu, G. Wen, D. Wu, Finite-time formation control for a group of quadrotor aircraft, Aerospace Science and Technology 69 (2017) 609-616.

[31] A. Levant, Homogeneity approach to high-order sliding mode design, Automatica 41 (5) (2005) 823-830.

[32] S. Wang, A. Polyakov, G. Zheng, On generalized homogenization of linear quadrotor controller, in: 2020 IEEE International Conference on Robotics and Automation (ICRA), IEEE, 2020, pp. 6190-6195.

[33] A. Polyakov, D. Efimov, W. Perruquetti, Robust stabilization of mimo systems in finite/fixed time, International Journal of Robust and Nonlinear Control 26 (1) (2016) 69-90.

[34] A. Mercado-Uribe, J. A. Moreno, A. Polyakov, D. Efimov, Integral control design using the implicit lyapunov function approach, in: 2019 IEEE 58th Conference on Decision and Control (CDC), IEEE, 2019, pp. 3316-3321.

[35] Y. Bouzid, H. Siguerdidjane, Y. Bestaoui, Sliding modes based nonlinear pid controller for quadrotor: Theory and experiment, in: 14th International Conference on Informatics in Control, Automation and Robotics (ICINCO 2017), Vol. 1, SCITEPRESS-Science and Technology Publications, 2017, pp. pages-286.

[36] S. Wang, A. Polyakov, G. Zheng, Quadrotor control design under time and state constraints: Implicit lyapunov function approach, in: 2019 18th European Control Conference (ECC), IEEE, 2019, pp. 650-655.

[37] Y. Orlov, Finite time stability and robust control synthesis of uncertain switched systems, SIAM Journal on Control and Optimization 43 (4) (2004) 1253-1271.

[38] E. Roxin, On finite stability in control systems, Rendiconti del Circolo Matematico di Palermo 15 (3) (1966) 273-282.

[39] S. Bouabdallah, Design and control of quadrotors with application to autonomous flying, Tech. rep., Epfl (2007).

[40] S. Bouabdallah, R. Siegwart, Full control of a quadrotor, in: 2007 IEEE/RSJ International Conference on Intelligent Robots and Systems, Ieee, 2007, pp. 153-158.

[41] P. S. Cisneros, C. Hoffmann, M. Bartels, H. Werner, Linear parameter-varying controller design for a nonlinear quad-rotor helicopter model for high speed trajectory tracking, in: 2016 American Control Conference (ACC), IEEE, 2016, pp. 486-491.

[42] R. Xu, U. Ozguner, Sliding mode control of a quadrotor helicopter, in: Proceedings of the 45th IEEE Conference on Decision and Control, IEEE, 2006, pp. 4957-4962.

[43] A. Polyakov, L. Hetel, C. Fiter, Relay control design using attractive ellipsoids method, in: 2017 IEEE 56th Annual Conference on Decision and Control (CDC), IEEE, 2017, pp. 6646-6651.

[44] A. F. Filippov, Differential equations with discontinuous righthand sides: control systems, Vol. 18, Springer Science \& Business Media, 2013.

[45] Y. Braidiz, D. Efimov, A. Polyakov, W. Perruquetti, On robustness of finite-time stability of homogeneous affine nonlinear systems and cascade interconnections, International Journal of Control (2020) 1-11. 